\title{
Preference for the light compartment of a light/dark cage does not affect rat exploratory behavior in the elevated plus-maze
}

\author{
Andrea Milena Becerra Garcia, Raquel Chacon Ruiz Martinez and Silvio Morato \\ Universidade de São Paulo, Ribeirão Preto, Brazil
}

\begin{abstract}
The are few reports on the relationship between elevated plus-maze and effects of maintenance conditions in the days prior to the test. Previously, we have demonstrated that being forcibly in total dark or in light for four days does not alter exploratory behavior in the plus-maze. The present study aimed at recording illumination level preferences in rats using a box with light and dark compartments (or another with two light compartments) and the behavioral effect of this choice on the plus-maze. The rats allowed to express their preference to remain in one particular level of illumination tended to stay initially in the dark compartment and gradually preferred to remain in the light compartment. When tested in the elevated plus-maze there were no effects in comparison to controls kept in commercial cages. Keywords: illumination level, preference for light or dark environments, elevated plus-maze, domestication, rats.
\end{abstract}

Received 24 April 2007; received in revised form 26 February 2008; accepted 29 February 2008. Available online 20 May 2008

\section{Introduction}

The elevated plus-maze, a modification inspired in a procedure introduced almost five decades ago by Montgomery (1955), was first reported by Handley \& Mithani (1984) and has often been used to investigate anxiolytic and anxiogenic compounds and to study the involvement of neurotransmitters in anxiety (e.g., Pellow \& File, 1986; Moser, 1989; Cruz, Frei \& Graeff, 1994; Rodgers \& Cole, 1994). The method was considered to be a valid and reliable tool for measuring anxiety, on the basis of extensive investigation analyzing several of its behavioral, physiological and pharmacological aspects (Pellow \& File, 1986; Cruz et al., 1994; Rodgers \& Cole, 1994; Anseloni \& Brandão, 1997). The test consists of placing an animal in a plus-shaped maze elevated above the floor level, with wall-closed arms and open arms, and measuring the frequency of entries and time spent in each type of arm as well as other behaviors not related to locomotion, such as rearing, stretching, etc. A rat explores both the closed and the open arms but will typically enter more frequently and stay longer in the

Andrea Milena Becerra Garcia, Raquel Chacon Ruiz Martinez and Silvio Morato Faculdade de Filosofia, Ciências e Letras de Ribeirão Preto, Universidade de São Paulo and Instituto de Neurociências e Comportamento, Ribeirão Preto, SP, Brazil. Correspondence concerning this article should be addressed to Silvio Morato, Faculdade de Filosofia, Ciências e Letras de Ribeirão Preto-USP, Av. Bandeirantes, 3900, Ribeirão Preto, SP, 14040-901. Phone: +55-16-36023662; Fax: +55-16-36024835. E-mail address: smorato@ffclrp.usp.br closed arms. The percent preference for open or closed arms, both for entries and time spent in them, is taken as an index of anxiety: the more intense the anxiety, the lower the percent preference for the open arms (e.g., Handley \& Mithani, 1984; Pellow \& File, 1986).

In spite of the apparent simplicity of this test situation, the aversion to the open arms seems to be influenced by many factors (for a review, see Carobrez $\&$ Bertoglio, 2005). Some of them are inherent to the subjects, such as sex (Johnston \& File, 1991; Imhof, Coelho, Schmitt, Morato, \& Carobrez, 1993) and age (Imhof et al., 1993). Others are linked to the experimental procedure, such as pre-exposure or even multiple exposures to the maze (File, 1992; Griebel, Moreau, Jenck, Martin, Misslin, 1993; Treit, Menard, \& Royan, 1993) and time of day at which testing occurs (Gentsch et al., 1982; Treit et al., 1993). Still others concern the test situation itself, such as the levels of illumination of the test room (Gentsch Lichtsteiner, Kraeuchi, \& Feer, 1982; Morato \& Castrechini, 1989; Griebel et al., 1993; Cardenas, Lamprea, \& Morato, 2001; Garcia, Cardenas, \& Morato, 2005). These studies indicate that exploration of the open arms is increased by low levels of environmental illumination. Also related to the test situation is the presence of raised edges surrounding the open arms, which prevent the rats from falling (Treit et al., 1993; Fernandez \& File, 1996; Cardenas et al., 2001), manipulation of experimental subjects, such as the way rats are transported to the test room (Morato \& Brandão, 1996), whether they are housed in groups or individually (Maisonnette, Morato, \& Brandão, 1993) or the place and how long they are kept there before testing (Morato \& Brandão, 1997). 
Despite the broad use of this animal model of anxiety, little is known about the event(s) triggering the aversion that ultimately cause rodents to avoid the open arms of the maze. It has been demonstrated that the natural aversion of rats to open spaces seems to be linked to rodent thigmotaxis (Treit et al., 1993) rather than to the fear of heights, as previously supposed (Pellow \& File, 1986). Cardenas et al. (2001) submitted rats to acute bilateral removal of mystacial vibrissae at different lengths from the follicle, interfering with the normal thigmotaxis. Their results showed that the vibrissal sense is not the main sensory modality in the exploration of the elevated plus-maze.

On the other hand, previous reports by Schiffman, Lore, Passafiume and Neeb (1970) suggest that, whenever possible, rats use vision as the main perceptual system to obtain relevant information from the environment. Jones and King (2001) have related that, when tested in the elevated plus-maze in the dark, rats showed increased locomotor activity without decreasing open-arm avoidance. Also, we have previously reported that rats tested under low intensity environmental light (20 lux) increased the number of entries and the time spent in open arms, as compared to rats that were tested under high intensity (1200 lux) environmental light (Morato \& Castrechini, 1989). In the same vein, Griebel et al. (1993) found that high illumination levels significantly reduced the percentage of entries into the open arms, the percentage of time spent there and total activity, in comparison to low illumination levels. They concluded that illumination has an effect on the exploratory behavior of rats in the elevated plus-maze. It seems, however, that illumination does not exert its effect in an intensity-dependent way. Garcia, Cardenas and Morato (2005), showed that when the animals are tested with illumination lower than 3 lux in the test room, there is increased open arm exploration. These results suggest that whenever possible, rats use vision as the main perceptual system to obtain relevant information from a novel environment, and that light is a more powerful trigger for anxiety than the absence of thigmotaxis, as already suggested by Cardenas et al. (2001).

Martinez, Cardenas, Lamprea and Morato (2002) investigated the role of transparent edges surrounding the open arms, which differed in height: 1, 5, 10, 20 and 40 $\mathrm{cm}$. Additionally, the $40-\mathrm{cm}$ high transparent edge was also studied covered with white translucent or black opaque paper. The rats avoided the open arms equally when those were surrounded by either $1-$ or $40-\mathrm{cm}$ high transparent edges (which allow thigmotaxis and surrounded the open arms as did the wooden walls). Also, this very same 40$\mathrm{cm}$ edge, when covered by the papers caused increases in exploratory behavior of the open arms (especially when the black paper was used), indicating that vision could trigger an aversion to open spaces causing the decreased exploratory behavior in the open arms.

It is important to note that all of the previously mentioned studies deal with the exploratory behavior of rats in unfamiliar environments (i.e. the animals being typically tested only once). In this respect, we previously demonstrated (Martinez, Garcia, \& Morato, 2005) that continuous exposure to a dark or light environment for 96 hours has no behavioral effect in the elevated plusmaze as compared to rats maintained under a 12/12 hour light/dark cycle. In this experiment, however, the lack of effect in the elevated plus-maze could be due to the fact that, in spite of being in a now-familiar environment, the animals could not choose the type of illumination they preferred. Thus, taking into consideration that rats seem not to be afraid of open spaces in the dark (as discussed in the preceding paragraphs), that they are nocturnal animals (Barnett, 1975) and that being able to choose the illumination level can be an important factor, we hypothesized that rats, if given the choice, prefer to be in the dark all the time. Also, we investigated whether the possibility to perform such a choice would affect behavior in the elevated plus-maze.

\section{Experiment I}

The present experiment was designed to test the hypothesis that, by being able to choose the illumination level in their home cages during daylight time, rats would prefer to be in the dark all of the time, a choice that could affect behavior in the elevated plus-maze. To achieve this goal, rats were kept in cages with two environments, which they could choose at any time: a dark covered or a light open one. We also studied the performance of the rats in the elevated plus-maze.

\section{Method}

\section{Subjects}

Thirty-six male Wistar-derived rats, weighting $200 \quad 10$ $\mathrm{g}$, were obtained from the animal house of the University of São Paulo at Ribeirão Preto. All the subjects were submitted to a habituation period of three days before the beginning of the experiment. The subjects were housed in groups of six per cage $(40 \times 34 \times 17 \mathrm{~cm})$ (Botelho, Estanislau, \& Morato, 2007). After this period, a group of control rats was kept in commercial polypropylene cages of the same size as the experimental groups of six throughout all the experiment. The two experimental groups, also kept in groups of six, are described in more detail in the Apparatus section. The animals were always kept in groups of six per cage (experimental or otherwise except during the behavioral test) under a 12/12 hour dark-light cycle (lights on at 7:00 a.m.). Room temperature was kept between 24$27^{\circ} \mathrm{C}$ and all subjects had free access to food and water. Behavioral testing always occurred between 8:00 and 11:30 a.m. The experiments reported in this paper were performed in compliance with the recommendations of the Brazilian Society of Neuroscience and Behavior which, in turn, are based on the U.S. National Institutes of Health Guide for Care and Use of Laboratory Animals.

\section{Apparatus}

After the three-day habituation period, the control rats (group C, $n=12$ ) were kept in commercial polypropylene cages covered with a metal grid, six to a cage. The grid had a depression in which food pellets and a bottle of water could be placed. In addition to those, we also used two boxes made of wood and lined with Formica. They were divided in the middle, length-wise by a wooden wall also lined with Formica, with an entrance $(8 \times 8 \mathrm{~cm})$ next to one of the walls. The boxes were designed so that each half was the same size and could be covered by the same commercial 
metal grid that usually covers the polypropylene cages. In the interior of one of the boxes, both compartments were lined with white Formica and covered with the commercial metal grids, thus forming two light compartments (group LL, $n=12$ ). The second box had one compartment lined with white Formica and was covered with the metal grid (light compartment) and another one lined with black Formica and was covered with a board (also lined with black Formica) that fit the borders of the compartment (dark compartment). During daylight, the animals kept in this box (group LD, $n$ $=12$ ) could choose to remain in the dark compartment or the light compartment, in the animal room 12-hour light/ dark cycle. The animals kept in the other box had no such a choice since both compartments were alike and subjected to the light regimen of the animal room. The position of both boxes in the animal room shelves were changed daily so that the entrance was one day near the vivarium wall and next day near the border of the shelf, away from the wall. Food pellets were placed inside both compartments near the dividing wall and away from the entrance; water bottles were placed only in one side, in the metal grid cover depression in both experimental cages.

For the behavioral tests an elevated plus-maze was used. It consisted of two open arms $(50 \times 10 \mathrm{~cm})$ crossed at right angles with two opposed arms of the same size. Two of the opposed arms were enclosed by walls $40 \mathrm{~cm}$ high, except for the central part where the arms crossed. The whole apparatus was elevated $50 \mathrm{~cm}$ above the floor. To prevent the rats from falling, a rim of Plexiglas $(1 \mathrm{~cm}$ high) surrounded the perimeter of the open arms. The experimental sessions were recorded with a video camera interfaced with a TV monitor and a VCR in an adjacent room. In order to record the number of crossings and other behaviors, the image of the elevated plus-maze on the TV screen was divided into $10-\mathrm{cm}$ squares. This allowed recording the number of squares entered by the animal as well as the exact place of occurrence of recorded behaviors.

\section{Procedure}

The presence of the rats in the compartments was recorded from 8:00 a.m. to 6:00 p.m. For the covered experimental box, we recorded how many rats were present in the light compartment at two hour intervals. In the box with two light compartments, the presence of rats was recorded only in the compartment in the same position as the light compartment of the other cage.

After four days, the rats in groups C, LL and LD were tested in the plus-maze under illumination (150 lux in the central square). Each rat was gently placed in the central area with the nose facing one of the closed arms and allowed to freely explore the maze for 5 minutes. Before the next rat was tested, the maze was cleaned with a $5 \%$ ethanol solution and dried with a cloth. The conventional measures (number of entries and time spent into the arms) were recorded. In addition, the frequency and time spent in the following behaviors was measured: (a) head dipping: dipping the head below the level of the maze floor; (b) stretching: when the animal stretches to its full length with the forepaws (keeping the hind paws in the same place) and turns back to the previous position.; (c) rearing: partial or total raising on the hind limbs; and (d) grooming: speciesspecific behavioral sequences including cleaning of any part of the body surface or fur with the tongue, teeth, and/ or paws (Cruz et al., 1994). The number of entries and the time spent in the distal parts (extremities comprising the two outer squares) of the open and closed arms as well as the total distance run in the arms (estimated from the number of squares entered) were also recorded.

\section{Statistical analysis}

At the end of each day, we recorded the total number of times each rat was seen in the light compartment at each 2-hour period. These presence data were averaged for each group and analyzed with two-way ANOVA, with type of box as one factor (two levels: groups LL and LD) and days as the repeated measure factor (four levels: days 1-4). The measurements obtained in the elevated plus-maze were analyzed using oneway ANOVA (three levels: groups C, LL and LD) and posthoc comparisons between groups, whenever necessary, were made using the Newman-Keuls multiple comparisons test. All tests were used with significance set at $p<0.05$.

\section{Results}

Figure 1 , shows the average (+SEM) of the number of rats in the 2-hour periods in the light compartment of the LD box (or its counterpart in the LL box) during the four experimental days. The ANOVA showed that there were differences due to the box type, $F(1,22)=4.93, p=0.04$, to the days, $F(3,66)=$ $6.98, p<0.001$, and showed a significant interaction between factors, $F(3,66)=6.73, p<0.001$. The Newman-Keuls test revealed that the mean number of observations of LL rats did not change in the four days. LD rats, on the other hand, significantly increased the mean number of observations in the light compartment in the third and fourth days, in comparison with the two first days. The mean number of observations in the first two days was smaller in group LD as compared to group LL.

Figure 2 shows the percentage entries into the open arms and the time spent in them (top) by the rats. The ANOVA showed no differences between the groups in the percentage of entries, $F(2,35)=0.13, p=0.88$, or in the time spent in the open arms, $F(2,35)=0.14, p=0.87$. Figure 2 also shows entries into the closed arms as well as the distance run in them (bottom). Again, the ANOVA showed no differences between groups in the entries into the closed arms, $F(2,35)=1.69, p=0.17$, or in the distance run in them, $F(2,35)=0.07, p=0.93$.

Table 1 shows the means ( \pm SEM) of the other behaviors recorded in the elevated plus-maze, as well as the ANOVA values of $F$ and $p$. The analysis did not show any differences in any of the behaviors.

\section{Discussion}

Data showed the rats exhibited a preference for remaining in the light compartment of the LD box during the daylight 


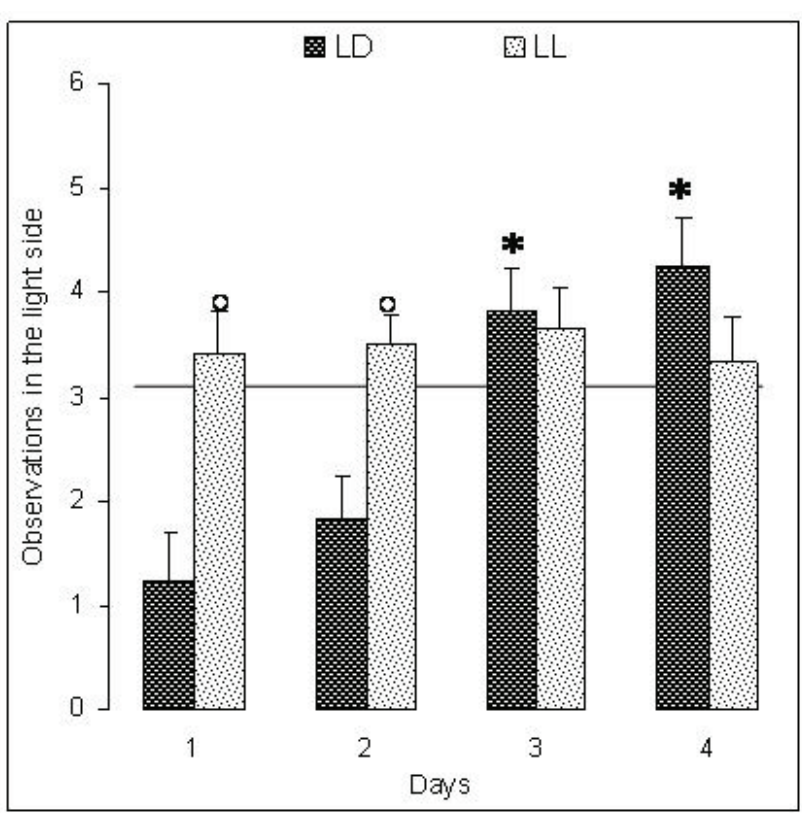

Figure 1. Number of rats present in the corresponding light side during the four days of LL or LD cages. Bars represent the mean and the vertical lines are SEMs. *, Significantly different from both days 1 and $2{ }^{\circ}{ }^{\circ}$, significantly different from animals in the LD group (Newman-Keuls, $p<0.05$ ).

hours, as indicated by the frequencies of recordings higher than that of the LL rats, which were always observed approximately half in one compartment and half in the other. This result indicates that the rats were not preferring the side based on where the food was, since food pellets were present in both compartments in each cage. The water bottle was placed only in the light compartment of the LD cage (or its counterpart in the LL cage) but this was not a factor in the choice of compartments since, as stated previously, half the rats in the LL cage chose the side containing the bottle but the other half chose the side without the bottle.

The preference for light exhibited by the rats in the LD cage, however, did not alter the measurements in the behavioral test. As far as the conventional anxiety measures - entries and time spent in open arms and their extremities - are concerned (Pellow, Chopin, File, \& Briley, 1985; Cruz et al., 1994), the groups housed in the different cages did not differ in terms of exploratory behavior in the elevated plus maze, in comparison to control rats kept in commercial cages. Also, there were no differences between the groups as far as the other behavioral categories related to anxiety - head dipping and stretching behaviors (Cole \& Rodgers, 1993; Anseloni \& Brandão, 1997) — are concerned. Nor were locomotion measures - entries into the closed arms and distance run in them, frequency and time spent rearing (File, 1992; Cruz et al., 1994) - affected, as judged by the absence of statistical differences between the groups. Thus, behavior in the elevated plus-maze is not at all affected by illumination level in the chosen compartment. This lack of behavioral effects in the plus-maze could be due to the fact that, in observational days 3 and 4, many animals in both groups (LL, LD) remained in a compartment where light was present during daytime, as was the case of the rats in the commercial cages.

The possibility of choosing the level of illumination by the rats kept in the LD cage, however, did affect their preference

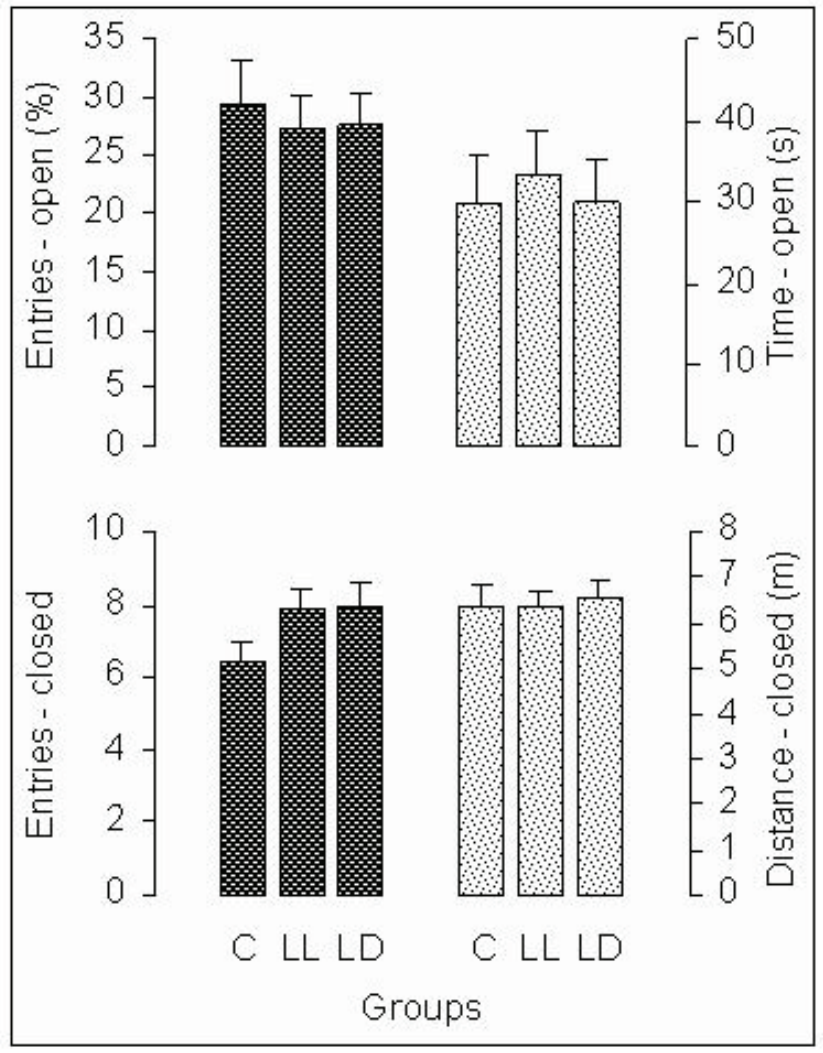

Figure 2. Exploratory behavior in the elevated plus-maze by the rats kept for four days in the different cages. Percentage of entries and time spent in the open arms (top); entries and distance run in the closed arms (bottom). C, control; LL, light-light cage; and LD, light-dark cage. Bars represent the means and vertical lines indicate SEMs.

for the light compartment. During the four days, the first group of rats in the LD cage gradually spent more time in the light compartment. Apparently, the animals stayed in the dark compartment only when the environment was still novel and, perhaps, potentially dangerous, which might have led them to choose this more protected area. This result is inconsistent with the nocturnal nature of the species (Barnett, 1975; Nasello Machado, Bastos, \& Felicio, 1998), which led us to the initial hypothesis that the rats would prefer to be mainly in the dark during daytime when given the chance.

As mentioned above, one possible explanation for the lack of behavioral effects in the plus-maze was that many LD rats spent the daytime under illuminated conditions, in similar amounts as control or LL rats, leading to the same performance in the elevated plus-maze. So, we designed Experiment II in order to investigate whether testing the animals in the second day in the LD cage, when the preference for the illuminated compartment was not yet established, would produce different behavioral data in the elevated plus-maze. The rationale underlying this hypothesis is that, up to the second day, most rats in the LD group still chose to remain in the dark compartment and thus would be in a different illumination condition during daytime than the rats in the C or LL groups. In this manner, we would test and compare rats that chose to remain in the dark during daytime against rats forced to be in the light in the LL and $\mathrm{C}$ groups, any differences being due to the light regimen.

Since the number of LD rats in the illuminated compartment was still increasing in the fourth day, it is 
Table 1. Means ( \pm SEM) of frequency and time spent in several behavioral parameters and their ANOVA statistical results.

\begin{tabular}{|c|c|c|c|c|c|}
\hline \multirow{2}{*}{ Parameters } & \multirow{2}{*}{$\frac{\text { Group C }}{\text { Mean SEM }}$} & \multirow{2}{*}{$\frac{\text { Group LI }}{\text { Mean SEM }}$} & \multirow{2}{*}{$\frac{\text { Group LD }}{\text { Mean SEM }}$} & \multicolumn{2}{|c|}{ ANOVA } \\
\hline & & & & $\mathrm{F}[2.35]$ & $\mathrm{P}$ \\
\hline Entries open arms & $2.8 \pm 0.4$ & $3.1 \pm 0.4$ & $3.1 \pm 0.4$ & 0.126 & 0.882 \\
\hline Mean duration - entries open arms (s) & $9.3 \pm 1.3$ & $10.4 \pm 0.8$ & $9.3 \pm 0.7$ & 0.456 & 0.638 \\
\hline Time spent in the open arm extremities & $10.2 \pm 3.1$ & $11.8 \pm 2.4$ & $11.3 \pm 2.5$ & 0.094 & 0.91 \\
\hline Time spent in the central square (s) & $28.3 \pm 4.1$ & $49.5 \pm 6.4$ & $43.3 \pm 9.6$ & 2.371 & 0.109 \\
\hline Frequency of stretching & $3.7 \pm 0.5$ & $4.3 \pm 0.6$ & $3.3 \pm 0.6$ & 0.778 & 0.468 \\
\hline Time spent stretching (s) & $6.7 \pm 1.1$ & $6.5 \pm 1.2$ & $4.9 \pm 0.9$ & 0.755 & 0.478 \\
\hline Frequency of rearing & $10.8 \pm 1,0$ & $12.3 \pm 1,0$ & $10.5 \pm 1.1$ & 0.822 & 0.448 \\
\hline Time spent rearing (s) & $20.4 \pm 2.1$ & $22.5 \pm 2.8$ & $20.4 \pm 2.4$ & 0.234 & 0.793 \\
\hline Frequency of grooming & $6.3 \pm 0.8$ & $5.9 \pm 0.8$ & $6.6 \pm 0.8$ & 0.172 & 0.843 \\
\hline Time spent grooming (s) & $37.0 \pm 7,0$ & $37.9 \pm 6.9$ & $32.1 \pm 4.3$ & 0.254 & 0.777 \\
\hline Frequency of head dipping & $7.6 \pm 1.1$ & $9.7 \pm 1.1$ & $9.1 \pm 1.3$ & 0.865 & 0.43 \\
\hline Time spent dipping head (s) & $14.6 \pm 2.3$ & $16.6 \pm 2.7$ & $16.3 \pm 2.3$ & 0.188 & 0.829 \\
\hline
\end{tabular}

possible that a larger number of rats could be recorded there if the animals remained longer in such a condition. Thus, in Experiment III, rats were studied for 12 days without being tested in the elevated plus-maze in order to ascertain the rats' preference for the dark or light side of the compartments.

\section{Experiment II}

The goal of this experiment was to investigate rats that could choose to remain in the dark during daytime hours but not so long as the rats could habituate to being in the illuminated compartment. Based on the data observed in Experiment I, on the second day preferences were not yet changed and that led us to test the rats in the plus-maze after this period of time and compare the results against rats forced to be in the light in the LL and $\mathrm{C}$ groups since any differences could be due to the light regimen.

\section{Method}

\section{Subjects}

Thirty-six male Wistar-derived rats $(20010 \mathrm{~g})$, with the same characteristics as in Experiment I, divided in three groups, also as in Experiment I (groups C, LL, LD). The only difference was that the rats remained in the experimental conditions for only 48 hours.

\section{Apparatus}

The same commercial and two-compartment cages and elevated plus-maze as described in Experiment I.

\section{Procedure}

The presence of rats in the light compartment was recorded as described in Experiment I, except that only for $48 \mathrm{~h}$. After this period, the animals were tested in the elevated plus-maze as in Experiment I.
Statistical analysis

The same as in Experiment I.

\section{Results}

Figure 3 shows the average (+ SEM) number of rats recorded in each 2-hour periods in the light compartment for the LD and LL cages along the two experimental days. The ANOVA showed that there were differences due to the box type, $F(1,22)=5.03, p=0.04$, but not to the experimental days, $F(1,22)=2.24, p=0.15$, and no significant interaction between factors, $F(1,22)=2.24$, $p=0.15$. The Newman-Keuls test revealed that the mean number of observations of LL rats did not change in the two days. LD rats, on the other hand, significantly increased the mean number of observations in the light compartment in the second day, in comparison with the first day. The mean number of observations in the LD group in the first day was significantly smaller than that of the LL group.

Figure 4 shows the percentage entries into the open arms and the time spent in them (top) by the rats in the first group, including the control group. The ANOVA showed no differences between the groups in the percentage of entries, $F(2,35)=2.39, p=0.68$, or in the time spent in the open arms, $F(2,35)=0.20, p=0.82$. Figure 4 also shows entries into the closed arms as well as the distance run in them (bottom). The ANOVA showed differences between groups in the entries into the closed arms, $F(2$, $35)=3.83, p=0.03$, but not in the distance run in them, $F(2,35)=1.688, p=0.20$. A post-hoc test showed an increase in the number of closed arm entries in the LL group when compared with the control group.

Table 2 shows the means ( \pm SEM) of the other behaviors recorded in the elevated plus-maze, as well as the ANOVA values of $F$ and $p$. The analyses showed differences in grooming and rearing frequency and 
stretching time. The post-hoc test showed a decreased in stretching time and grooming frequency in the LD group when compared with the control group. The test also showed increased rearing frequency in the LL and LD groups in comparison with the control group.

\section{Discussion}

As observed in Experiment I, the possibility of choosing the illumination condition in the animal room during daytime did not significantly alter the performance exhibited by rats in the elevated plus-maze. Despite the fact that these rats increased the time spent in the illuminated compartment (while LL rats occupied both compartments equally) in the second day, this preference did not

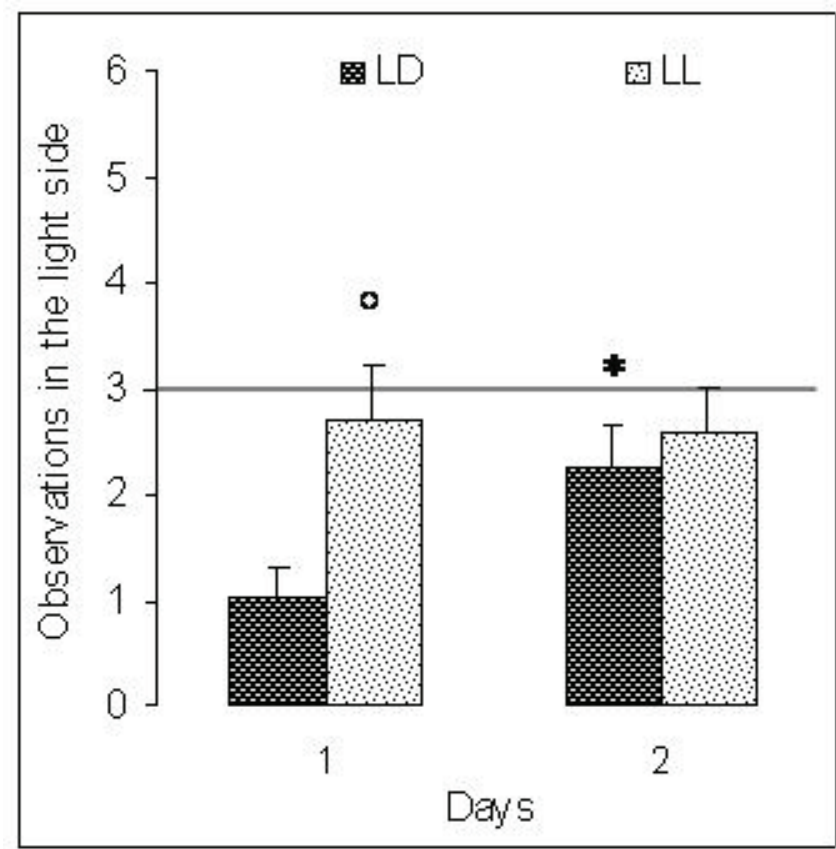

Figure 3. Number of rats present in the corresponding light side during the two days of LL or LD cages. Bars represent the mean and the vertical lines are SEMs. *, Significantly different from day $1 ;{ }^{\circ}$, significantly different from animals in the LD group (Newman-Keuls, $p<0.05$ ).

significantly alter the behavior exhibited in the elevated plus-maze test. As in Experiment I, the preference for the dark compartment during the two days before testing did not alter conventional anxiety measures - entries and time spent in open arms - or exploration measures such as entries into the closed arms or the distance run in them. Finally, the LD rats decreased stretching time and grooming frequency and increased rearing frequency. These changes are compatible with a slight decrease in anxiety, not enough to affect the conventional measures but enough to alter other measures.

The hypothesis that LD rats, by remaining more in the dark compartment in the first two days, would exhibit different exploratory patterns in the elevated plus-maze was not supported. Thus, the possibility of choosing a dark environment during daylight hours is not a factor that affects exploratory behavior in novel environment nor the anxiety or fear that accompanies such exploration.

\section{Experiment III}

The aim of the present experiment was to observe rats in the LD cage to determine when the animals start exhibiting a preference for the illuminated compartment. We chose to study the rats for a period of 12 days, since the four days of observation in Experiment I seemed not enough for the full preference to appear.

\section{Method}

\section{Subjects}

Twelve male Wistar-derived rats $\left(\begin{array}{lll}200 & 10 \mathrm{~g}\end{array}\right)$ with the same characteristics as those of Experiments I and II were used. After the habituation period, the subjects

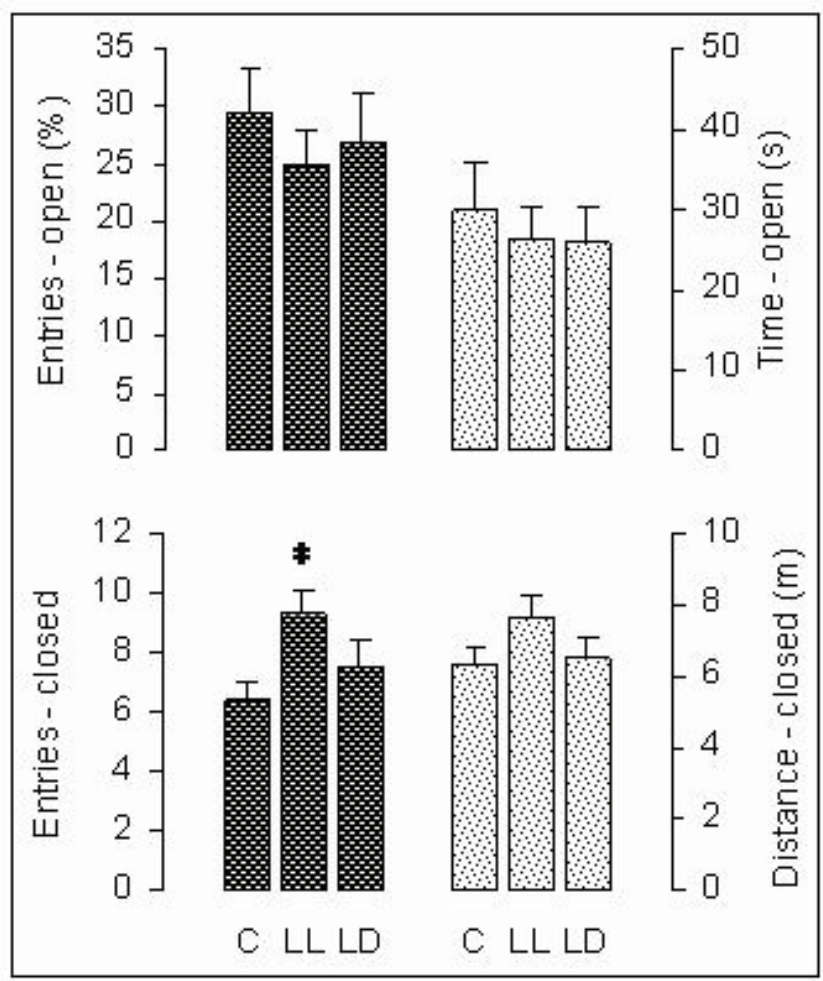

Figure 4. Exploratory behavior in the elevated plus-maze by the rats kept for two days in the different cages. Percentage of entries and time spent in the open arms (top); entries and distance run in the closed arms (bottom). C, control; LL, light-light cage; and LD, light-dark cage. Bars represent the means and vertical lines indicate SEMs. *, Significantly different from C.

were housed for 12 days in the same two-compartment cages and conditions used in Experiments I and II (groups LL, LD).

\section{Apparatus}

The same two-compartment cages described in Experiments I and II.

\section{Procedure}

The number of rats present in the light compartment was recorded every two hours, from 08:00 $\mathrm{h}$ to 18:00 $\mathrm{h}$, as described in Experiments I and II during, for 12 days. 
Table 2. Means ( \pm SEM) of frequency and time spent in Experiment II for several behavioral parameters and their ANOVA statistical results. *,different from Group C. (Newman-Keuls, $\mathrm{p}<.05$ )

\begin{tabular}{|c|c|c|c|c|c|}
\hline \multirow{2}{*}{ Parameters } & \multirow{2}{*}{$\frac{\text { Group C }}{\text { Mean SEM }}$} & \multirow{2}{*}{$\frac{\text { Group LL }}{\text { Mean SEM }}$} & \multirow{2}{*}{$\frac{\text { Group LD }}{\text { Mean SEM }}$} & \multicolumn{2}{|c|}{ ANOVA } \\
\hline & & & & $\mathrm{F}[2.35]$ & $\mathrm{P}$ \\
\hline Entries open arms & $2.8 \pm 0.4$ & $3.1 \pm 0.5$ & $3,0 \pm 0.6$ & 0.069 & 0.933 \\
\hline Mean duration - entries open arms (s) & $9.3 \pm 1.3$ & $9,0 \pm 1,0$ & $8.2 \pm 0.9$ & 0.27 & 0.765 \\
\hline Time spent in the open arm extremities & $10.2 \pm 3.1$ & $10.4 \pm 2.1$ & $9.4 \pm 2.3$ & 0.513 & 0.95 \\
\hline Time spent in the central square (s) & $28.3 \pm 4.1$ & $41.2 \pm 4.1$ & $34.2 \pm 4.2$ & 2.453 & 0.102 \\
\hline Frequency of stretching & $3.7 \pm 0.5$ & $3.4 \pm 0.6$ & $1.9 \pm 0.5$ & 3.270 & 0.051 \\
\hline Time spent stretching ( $s$ ) & $6.7 \pm 1.1$ & $5,0 \pm 0.9$ & $2.6 \pm 0.7^{*}$ & 4.781 & 0.015 \\
\hline Frequency of rearing & $10.8 \pm 1,0$ & $14.4 \pm 1.1$ & $14.6 \pm 1.3^{*}$ & 3.732 & 0.035 \\
\hline Time spent rearing (s) & $20.4 \pm 2.1$ & $24.1 \pm 2,0$ & $25.7 \pm 2.8$ & 1.335 & 0.277 \\
\hline Frequency of grooming & $6.3 \pm 0.8$ & $3.8 \pm 0.6$ & $3.1 \pm 0.5^{*}$ & 6.754 & 0.003 \\
\hline Time spent grooming (s) & $37,0 \pm 7,0$ & $32.2 \pm 5.6$ & $22.2 \pm 6.4$ & 1.412 & 0.258 \\
\hline Frequency of head dipping & $7.6 \pm 1.1$ & $10.9 \pm 1.1$ & $10.1 \pm 1.2$ & 2.399 & 0.106 \\
\hline Time spent dipping head (s) & $14.6 \pm 2.3$ & $20.2 \pm 2,0$ & $17.8 \pm 1.9$ & 1.825 & 0.177 \\
\hline
\end{tabular}

\section{Statistical analysis}

The data were averaged in four three-day blocks for each rat and then analyzed with a two-way ANOVA, with type of box as one factor (two levels: cages LL and LD) and days as the repeated measure factor (four levels: blocks 1-4). Whenever necessary, post-hoc comparisons were performed using the Newman-Keuls multiple comparisons test. All tests were used with significance set at $p<0.05$.

\section{Results}

Figure 5 shows the average (+ EPM) of the number of rats observed in each2-hourperiod in the light compartment of the LD cage (or its counterpart in the LL cage) along the four three-day blocks. The ANOVA showed that there were differences due to box type, $F(1,10)=32.23$, $p<0.001$, but not to the blocks, $F(3,30)=0.40, p=0.75$, and showed a significant interaction between factors, $F(3,30)=11.92, p<0.001$. The Newman-Keuls test revealed that, as expected, LL rats did not differ along the four blocks, while LD rats were recorded in larger numbers in blocks 3 and 4 as compared to blocks 1 and 2 or the LL rats.

\section{Discussion}

The data indicated that when rats have a choice between remaining in dark or illuminated compartments during daytime, they gradually prefer to remain in the illuminated one. This probably indicates that the preference for light comes gradually, as the environment becomes familiar. The rats in the two-compartment illuminated cage did not have such a choice and thus approximately half of the group remained in each compartment, indicating that, given two compartments with similar characteristics, rats tend to occupy both environments in a similar way, as opposed to grouping in only one compartment. Thus, familiarity with the environment seems to be the important factor in choosing the illuminated side of the cage.

\section{General Discussion}

Our results show a gradual increase in the preference for the light compartment by the rats during the light period of the day. This increased preference for the light compartment is difficult to explain, especially in view of the nocturnal characteristic of a species that, in the wild, usually remains in dark burrows during the daylight period (Barnett, 1975; Nasello et al., 1998). These non-domesticated rats have a nocturnal nature, going out during the night and coming back before the beginning of the day (Barnett, 1975). As a possible explanation, one can consider that the rats we used descended from a long lineage of rats reared by man, which could induce some loss of the original behavioral characteristics of the ancestor wild species, such as an aversion to light. Such a relatively fast change in important biochemical characteristics have already been reported to occur in less than fifty generations (Lemos, Zucoloto, \& Terra, 1992; Zucoloto, 1993). A more prosaic alternative is that, by being reared in vivaria under light/dark cycles since a very early age, the animals got used to being in the light. Another explanation involves habituation to the place: the continuous housing under the new environmental conditions could, by the lack of aversive stimuli in the long run, render the light compartment of the LD cage as safe as the dark compartment, consequently allowing the rats to remain in it. So it remains that, instead of being repelled by illumination, the rats were actually attracted to it. Whether domestication or habituation (plus lack of aversive stimuli) is explanatory for such a preference for illumination can only be determined by experiments specifically addressed to this question. 


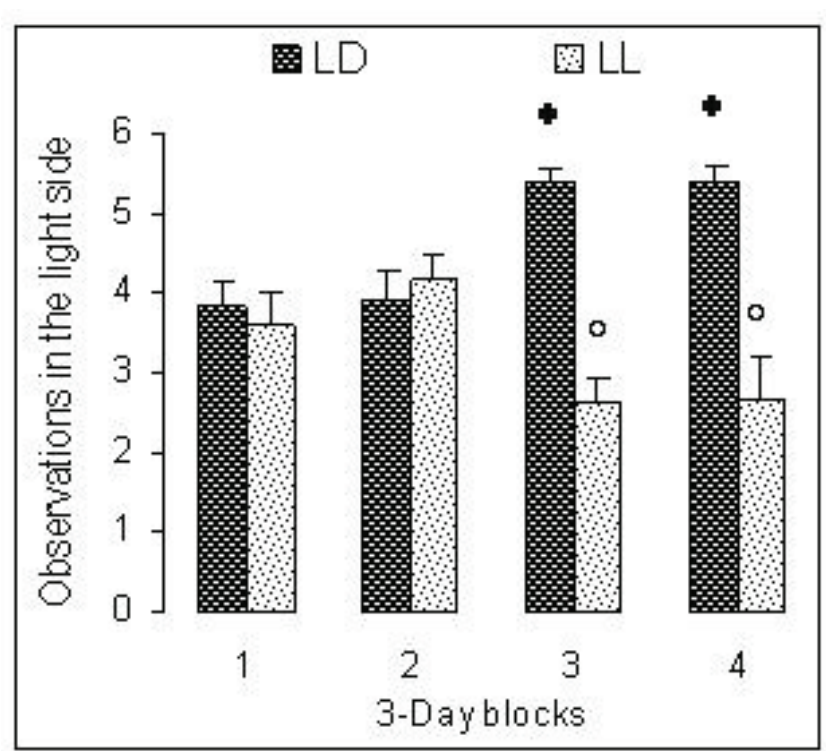

Figure 5. Number of rats present in the corresponding light side during the twelve days grouped in four 3-day blocks of LL or LD cages. Bars represent the mean and the vertical lines are SEMs. *, Significantly different from block 1 and $2 ;^{\circ}$, significantly different from animals in the LD group (Newman-Keuls, $\mathrm{p}<0.05$ ).

\section{Acknowledgment}

This research was supported by a research grant to SM (351317/96-1), from CNPq; RM and AMBG were the recipients of fellowships from $\mathrm{CNPq}$ and CAPES, respectively.

\section{References}

Anseloni, V.Z., \& Brandão, M.L. (1997). Ethopharmacological analysis of behavior of rats using variations of the elevated plusmaze. Behavioral Pharmacology, 8, 533-540.

Barnett, S.A. (1975). The Rat: A Study in Behavior. The University or Chicago Press Chicago.

Becker, A., \& Grecksch, G. (1996). Illumination has no effect on rats' behavior in the elevated plus-maze. Physiology \& Behavior, 59,175-177.

Bothelho, S., Estanislau, C., \& Morato, S. (2007). Effects of underand overcrowding on exploratory behavior in the elevated plusmaze. Behavioural processes, 74, 357-362.

Cardenas, F., Lamprea, M.R., \& Morato, S. (2001). Vibrissal sense is not the main sensory modality in the rat exploratory behavior in the elevated plus-maze. Behavioural Brain Research, 122, 169-174

Carobrez, A.P., \& Bertoglio, L.J. (2005). Ethological and temporal analyses of anxiety-like behavior:The elevated plus-maze model 20 years on, Neuroscience and Biobehavioral Reviews, 29, 11931205.

Cole, J.C., \& Rodgers, R.J. (1993). An ethological analysis of the effects of chlordiazepoxide and bretazenil (Ro 16-6028) in the murine elevated plus-maze, Behavioral Pharmacology, 4, 573-580.

Cruz, A.P.M., Frei, F., \& Graeff, F.G. (1994). Ethopharmacological analysis of rat behavior on the elevated plus-maze. Pharmacological Biochemistry and Behavior, 49, 171-176.

Fernandez, C., \& File, S.E. (1996). The influence of open arm ledges and maze experience in the elevated plus maze. Pharmacological Biochemistry and Behavior, 54, 31-40.

File, S.E. (1992). Behavioural detection of anxiolytic action In: J.M. Elliott D.J. Heal and C.A. Marsden. Experimental Approaches to Anxiety and Depression, New York. John Wiley and Sons Ltd, 25-44.
Garcia A.M.B., Cardenas F.P., \& Morato S (2005) Effect of different illumination levels on rat behavior in the elevated plus-maze. Physiology and Behavior, 85, 265-70

Gentsch, C., Lichtsteiner, M., Kraeuchi, K., \& Feer, H. (1982). Different reaction patters in individually and socially reared rats during exposures to novel environments. Behavioral Brain Research, 4, 45-54.

Griebel, G., Moreau, J.L., Jenck, F., Martin, J.R., \& Misslin, R. (1993). Some critical determinants of the behaviour of rats in the elevated plus-maze. Behavioral Processes, 29, 37-48.

Handley, S.L., \& Mithani, S. (1984). Effects of alpha-adrenoceptor agonists and antagonists in a maze-exploration model of 'fear'motivated behaviour. Naunyn-Schmiedeberg's Archives of Pharmacology, 327, 1-5.

Hogg, S. (1996). A review of the validity and variability of the elevated plus-maze as an animal model of anxiety. Pharmacological Biochemistry and Behavior, 54, 21-30.

Imhof, J.T., Coelho, Z.M.I., Schmitt, M.L., Morato, G..S., \& Carobrez, A.P. (1993). Influence of gender and age on performance of rats in the elevated plus-maze apparatus. Behavioral Brain Research, 56, 177-180.

Johnston, A.L., \& File, S.E. (1991). Sex differences in animal tests of anxiety. Physiology and Behavior, 49, 245-250.

Jones, N., \& King, S.M. (2001). Influence of circadian phase and test illumination on pre-clinical models of anxiety. Physiology and Behavior, 72, 99-106.

Lemos, F.J.A., Zucoloto, F.S., \& Terra, W.R. (1992). Enzymological and excretory adaptations of Ceratitis-capitata (Diptera, Tephritidae) larvae to high protein and high salt diets. Comparative Biochemistry and Physiology A-Physiology, 102 , 775-779.

Maisonnette, S., Morato, S., \& Brandão, M.L. (1993). Role of resocialization and of 5-HT1A receptor activation on the anxiogenic effects induced by isolation in the elevated plus-maze test. Physiology and Behavior, 54, 753-758.

Martinez, J.C., Cardenas, F., Lamprea, M., \& Morato, S. (2002). The role of vision and proprioception in the aversion of rats to the open arms of an elevated plus maze. Behavioral Processes, 60, 15-26.

Martinez, R., Garcia, A.M.B., \& Morato, S. (2005). Papel da luminosidade do biotério no comportamento do rato no labirinto em cruz elevado. Estudos de Psicologia, 10, 239-245.

Montgomery, K.C. (1955). The relation between fear induced by novel stimulation and exploratory behavior. Journal of Comparative and Physiological Psychology. 48, 254-260.

Morato, S., \& Brandão, M.L. (1996). Transporting rats to the test situation on a cart can modify rat exploratory behavior in the elevated plus-maze. Psychobiology, 24, 247-252.

Morato, S., \& Brandão, M.L. (1997). Paradoxical increase of exploratory behavior in the elevated plus-maze by rats exposed a two kinds of aversive stimuli. Brazilian Journal Of Medical And Biological Research, 30, 1113-1120.

Morato, S., \& Castrechini, P. (1989). Effects of floor and environmental illumination on exploratory activity in the elevated plus-maze, Brazilian Journal Of Medical And Biological Research, 22, 707-710.

Moser, P.C. (1989). An evaluation of the elevated plus maze test using the novel anxiolytic buspirone. Psychopharmacology, 99, 48-53.

Nasello, A.G., Machado, C., Bastos, J.F., \& Felicio, L.F. (1998). Sudden darkness induces a high activity-low anxiety state in male and female rats. Physiology and Behavior, 63, 451-454.

Pellow, S., \& File, S.E. (1986). Anxiolytic and anxiogenic drug effects on exploratory activity in elevated plus-maze: a novel test of anxiety in the rat, Pharmacology Biochemical and Behavior, 24, 525-529.

Pellow, S., Chopin, P., File, S.E., \& Briley, M. (1985). Validation of open: closed arm entries in an elevated plus-maze as a measure of anxiety in the rat. Journal of Neuroscience Methods, 14, 149-167.

Rodgers, R.J., \& Cole, J.C. (1994). The elevated plus-maze: Pharmacology methodology and ethology In: S.J. Cooper and C.A. Hendrie (Ed.), Ethology and Psychopharmacology. (pp. 9-43). New York, John Wiley and Sons Ltd,.

Schiffman, H.R., Lore, R., Passafiume, J., \& Neeb, R. (1970). Role of vibrissae for depth perception in the rat (Rattus novergicus). Animal Behaviour, 18, 290-292.

Treit, D., Menard, J., \& Royan, C. (1993). Anxiogenic stimuli in the elevated plus-maze. Pharmacology biochemistry and behavior, 44, 463-469.

Zucoloto, F.S. (1993). Adaptation of a Ceratitis-capitata population Diptera, Tephritidae to an animal protein-based diet. Entomologia Experimentalis et Applicata, 67, 119-127. 